\title{
Human-Scale Virtual Environment for Product Design: Effect of Sensory Substitution
}

\author{
Paul Richard, Damien Chamaret, François-Xavier Inglese, Philippe Lucidarme, Jean-Louis Ferrier
}

\begin{abstract}
This paper presents a human-scale virtual environment (VE) with haptic feedback along with two experiments performed in the context of product design. The user interacts with a virtual mock-up using a large-scale bimanual string-based haptic interface called SPIDAR (Space Interface Device for Artificial Reality). An original self-calibration method is proposed. A vibro-tactile glove was developed and integrated to the SPIDAR to provide tactile cues to the operator. The purpose of the first experiment was: (1) to examine the effect of tactile feedback in a task involving reach-and-touch of different parts of a digital mock-up, and (2) to investigate the use of sensory substitution in such tasks. The second experiment aimed to investigate the effect of visual and auditory feedback in a car-light maintenance task. Results of the first experiment indicate that the users could easily and quickly access and finely touch the different parts of the digital mock-up when sensory feedback (either visual, auditory, or tactile) was present. Results of the of the second experiment show that visual and auditory feedbacks improve average placement accuracy by about $54 \%$ and $60 \%$ respectively compared to the open loop case.
\end{abstract}

Index Terms - Virtual reality, virtual environment, haptic interaction, sensory substitution, human performance.

\section{INTRODUCTION}

According to their sizes, we could divide virtual environments (VEs) into two categories: small-scale or desk-top VEs and human-scale VEs. Desk-top VEs include all situations where the user is sitting still in front of a desktop monitor or wearing a Binocular Omni-Orientation Monitor (BOOM) [1]. These kinds of VEs generally constrain user movements within a small workspace. In large-scale assembly simulations, the operator needs to operate and interact with virtual objects in a large workspace. This lead to some challenges concerning human-scale haptics (kinesthetic and tactile).

Both accessibility testing and assembly simulations are interactive processes involving the operator and the handled objects, and hence simulation environments must be able to react according to the user's actions. Furthermore, the action of the user and the reaction of the virtual world must be presented in an intuitively comprehensible way. Therefore, it is of great importance to investigate the factors related to information

Manuscript received September 4, 2006. This work was supported in part by the Institut des Sciences et Techniques de l'Ingénieur d'Angers (ISTIA) and the Laboratoire d'Ingénierie des Systèmes Automatisés (LISA).

Paul Richard is with the LISA, 62 av. ND du Lac 49000 Angers (phone: 241-226-547; fax: 241-226-561; e-mail: paul.richard@univ-angers.fr).

Damien. Chamaret is with LISA (e-mail: chamaret@istia.univ-angers.fr). François-Xavier Inglese is with LISA (e-mail: inglese@istia.univ-angers.fr). Philippe Lucidarme is with LISA (e-mail: lucidarme@univ-angers.fr). Jean-Louis Ferrier is Director of LISA (e-mail: ferrier@istia.univ-angers.fr). presentation modalities that affect human performance in such tasks. However, despite some efforts in human-scale simulation, limited research has been performed to investigate the effects of isolated or multi-sensory feedbacks such as auditory, visual and haptic feedback on task performance. The experiments described in this article contribute to this research issue.

This paper is organized as follows: in Section 2, we describe and compare haptic interfaces, from desktop to human-scale. Then, in Section 3 we describe VIREPSE (Virtual Reality Platform for Simulation and Experimentation), a human-scale VE with haptic feedback that we have developed along with an original self-calibration method for the SPIDAR. In Section 4, we present two applications that have been integrated to VIREPSE. Sections 5 and 6 present the experiments that have been carried out. A conclusion is given in Section 7 .

\section{HAPTIC INTERFACES}

Haptic interfaces are robotic devices that enable manual interactions with VEs or tele-operated remote systems, using the sense of force-feedback. A survey of the haptic interface devices developed so far can be found in [2,3,4,5]. Most of these haptic interfaces are used for industrial applications in which immersion and presence is not crucial. Indeed, they are intrusive, expensive and have a limited workspace [6]. Moreover, although many industrial tasks are bimanual, these interfaces involve the use of only one hand $[6,7,8]$.

Attempts to add force feedback to large-scale virtual environments have been proposed such as one from the UNC [9, 10] and work from the University of Utah [11]. Both approaches are in fact quite similar. Both employs a one-screen workbench and propose installing an arm-type force feedback device. More recently, Lécuyer et al. experimented with a portable haptic device, which could follow large-scale user's displacements in front of a two-screen workbench [12]. This interface called the Wearable Haptic Handle (W2H), developed by CEA, is made of two parts. The upper part is a small platform, which moves in 6 Degrees of Freedom (DoF) actuated by a wire driven based Stewart platform. The user feels the displacements of the platform through his/her hand while interacting with the virtual environment. The $\mathrm{W} 2 \mathrm{H}$ has a wide workspace, which can match the large visualization space of the workbench and is small enough not to obstruct the user's field of view. A floor-grounded haptic device for aircraft engine maintainability (LHIfAM) has been recently developed [13]. This device is used to track hand movement and provides force feedback within a large workspace. Other kinds of haptic interfaces are currently being evaluated for education, entertainment and industrial applications [14, 15, 16, 17, 18]. These alternative interfaces are composed of actuators providing a force through a set of strings adequately linked 
together or to a manipulation tool [19, 20, 21, 22, 23, 24, 25]. A quick look at such interfaces shows that most of them have very interesting properties i.e. fixed-base, large workspace, and low intrusion. Additional properties like lightness, safeness, or low cost are also satisfied. However, these interfaces are complex to set-up and not easy to control. Paljic and Coquillart proposed a passive stringed-based haptic feedback system that can provide the user with grounded forces in a 3D manipulation space [26]. This interface uses brakes instead of motors and is easier to control, but it cannot simulate variable force feedback and therefore interaction with deformable virtual objects.

\section{VIREPSE}

This section presents the human-scale VE called VIREPSE that provides force feedback using the SPIDAR system (Space Interface Device for Artificial Reality) invented by Professor Makoto Sato at the Tokyo Institute of Technology [16]. Stereoscopic images are displayed on a rear-projected large screen $(2 \mathrm{~m} \times 2.5 \mathrm{~m})$ and are viewed using polarized glasses. A 5.1 immersive sound system and olfactory displays are used for simulation realism, sensorial feedback and immersion.

The SPIDAR system uses a SH4 controller from the Cyverse Inc. and Springhead physic engine [27]. In order to provide force feedback to both hands, a total of 8 motors are placed on the corners of a cubic frame surrounding the user (Fig. 1a). The system uses RE10 DC motors from Maxon Inc. [28], pulleys and optical encoders (Fig. 1b). Each end of the hand attachment is wrapped around a pulley driven by a DC motor. As illustrated in Fig. 2a, by controlling the tension and length of each string, the system generates appropriate forces for both hands.

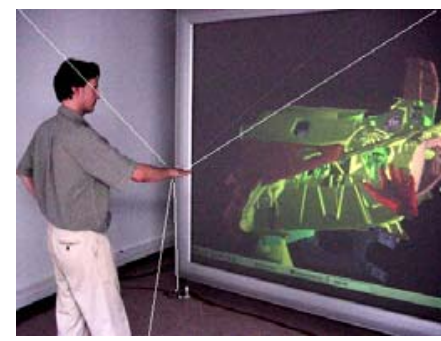

(a)

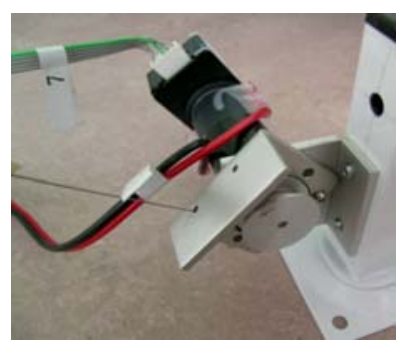

(b)
Fig. 1: (a) Operator using the VIREPSE human-scale stringed-based haptic interface, (b) motor, pulley and encoder.

\section{A-System workspace}

VIREPSE workspace could be divided into two spaces: (i) reachable space that gathers every point users can reach with hands [17], and haptic space that gathers every point where the system can produce a force in any direction. The global workspace is defined by the intersection of these two spaces. The workspace of the reachable space matches the cubic frame of the SPIDAR. Haptic spaces are illustrated on Fig. 2b (left hand) and 2c (right hand). Motors positioned at corners 1-3-6-8 are used to display forces on the user's right hand while motors positioned at corners 2-4-5-7 are used to display forces on the user's right left. Note that the haptic space is described by a tetrahedron. This shape is a theoretical workspace; in practice the real workspace is smaller than this shape. Besides, the faces of the tetrahedron are not included in the workspace. It seems natural that when the position of the hand attachment is located on the face, a force cannot be produced in any direction outside the space. The principle is that the closer the hand attachment is to the center of the tetrahedron, the more efficient the system becomes (it can produce a significant force in any direction).

\section{$B$ - Position measurement}

Let the coordinates of the hand attachment position be $P(x, y, z)$, which represent both the hand position, and, $l_{i}$, the length of the $i^{\text {th }}$ string $(i=0,1,2,3)$. To simplify the problem, let the four actuators (motor, pulley, encoder) be on four non-adjacent vertices of the cubic frame, as shown in Fig. $2 b$. Then $P(x, y, z)$ must satisfy equations $(1) \sim(4)$.

$$
\begin{aligned}
& l_{0}{ }^{2}=(x+a)^{2}+(y+a)^{2}+(z+a)^{2} \\
& l_{1}{ }^{2}=(x-a)^{2}+(y-a)^{2}+(z+a)^{2} \\
& l_{2}{ }^{2}=(x-a)^{2}+(y+a)^{2}+(z-a)^{2} \\
& l_{3}{ }^{2}=(x+a)^{2}+(y-a)^{2}+(z-a)^{2}
\end{aligned}
$$

The length of the cube is 2a. After some mathematical manipulations, we can obtain the position of a hand attachment as the following equation (5), in function of the lengths $l_{i}$ :

$$
\left\{\begin{array}{l}
x=\frac{\left(l_{0}{ }^{2}-l_{1}{ }^{2}-l_{2}{ }^{2}+l_{3}{ }^{2}\right)}{8 a} \\
y=\frac{\left(l_{0}{ }^{2}-l_{1}{ }^{2}+l_{2}{ }^{2}-l_{3}{ }^{2}\right)}{8 a} \\
z=\frac{\left(l_{0}{ }^{2}+l_{1}{ }^{2}-l_{2}{ }^{2}-l_{3}{ }^{2}\right)}{8 a}
\end{array}\right.
$$

The coordinates' origin is set at the center of the framework. The position measurement (through measurements of $l_{i}$ 's) ranges for all $x, y$, and $z$ within $[-1.25 \mathrm{~m},+1.25 \mathrm{~m}]$. Inside the position measurement range, the absolute static position measurement error is less than $0.6 \%$ of the workspace. The bandwidth is about $10 \mathrm{KHz}$. The maximum exerted force is $30 \mathrm{~N}$.

\section{C - Force control}

The system uses the resultant force of tension from strings to provide force display. The hand attachment is suspended by four strings, giving certain tensions to each of them by means of motors. The resultant force occurs at the position of the hand attachment, where it is transmitted to and felt by the operator'shand. Let the resultant force be $\vec{f}$ and the unit vector of the tension be $\vec{u}_{i}$ (i=0,1, 2, 3), the resultant force is given by equation 6 . 
The International Journal of Virtual Reality, 2006, 5(2):37-44

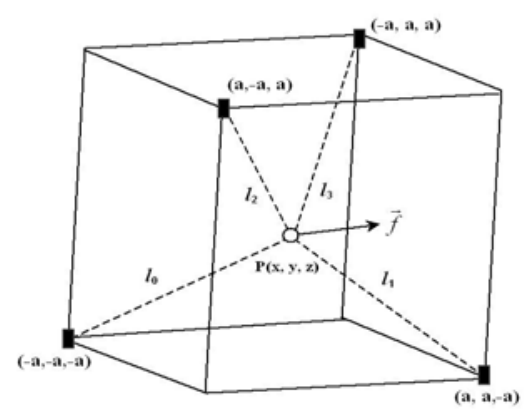

(a)

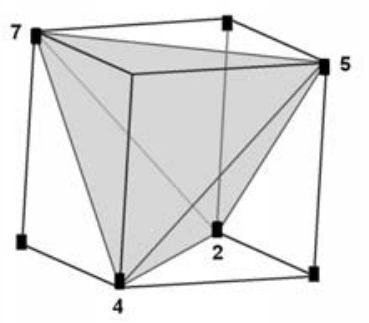

(b)

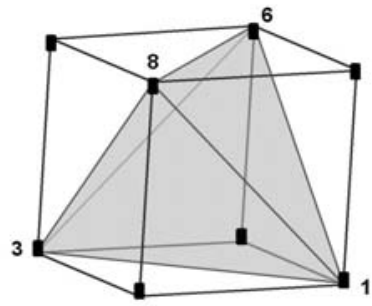

(c)
Fig. 2: (a) Position measurement and resultant force within the cubic framework of the SPIDAR; Haptic workspace (tetrahedron): (b) for right hand and (c) for left hand.

$$
\vec{f}=\sum_{i=0}^{3} k_{i} \overrightarrow{u_{i}} \quad k_{i}>0
$$

where $k_{i}$ represents the tension value of each string. By controlling all of the $k_{i}$, a resultant force in any direction can be composed.

\section{D-Calibration method}

Calibration generally requires an external measurement device that provides the absolute position of the end-effector of the robot. Unlike robots, the SPIDAR can be calibrated without external system according to the following hypothesis:

- The parameters to calibrate are the position of each motor.

- The exact length of each string is known at any time.

- The strings are long enough to reach any motor.

- The hand attachment is considered as a point.

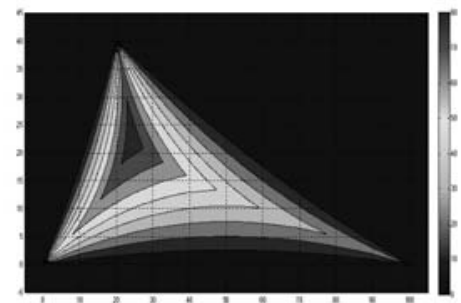

(a)

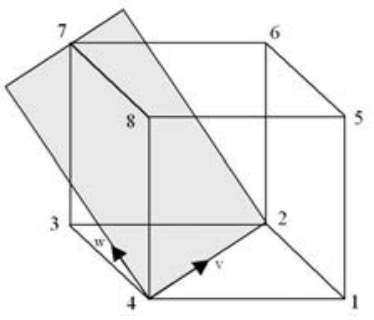

(b)
Fig. 3: (a) Performance of the device at any location inside the given workspace; (b) Position and orientation of the original frame used in the calibration method.
Let's first consider a 2D device equipped with 3 motors located at the positions $(0,40),(20,40),(100,0)$. We assume that the three motors are identical. As explained before, the haptic space and the reachable space are merged, thus the global space is given by the triangle formed by the three motors. Let's now examine the haptic workspace. When the hand attachment is on the edge of the triangle, the device cannot exert a force toward outside of the workspace. It means that the edges of the triangle are not included in the workspace. Fig. 3a shows the performance of the device at any location inside the workspace. For example, in the red area (second smaller area), the performance reaches eighty percent, i.e. if a motor is able to exert a force of $F \mathrm{~N}$ on a string, the device can only exert a maximum force of $0.8 F \mathrm{~N}$ in all directions in this region. Moreover, as the hand attachment moves away from the central area, the performance decreases. To avoid this problem and increase the system efficiency, one must add one or several motors; however, this also increases system complexity.

In order to calibrate the SPIDAR, it is necessary to set the position and orientation of an original frame. We have no guaranty that the motors are placed exactly on the corner of the cube, so, taking the cube as original frame may be a wrong hypothesis. We will define a new frame independent from the cube. As shown in Fig. 3(b), the origin of the frame is located on Motor 4. The $v$ axis is given by the vector Motor 4 -Motor2, and the plan $v$-w passes through Motor 7.

According to the previous relationships, the position of each motor can be defined by:

Motor $2:\left[\begin{array}{c}0 \\ v_{2} \\ 0\end{array}\right] \quad$ Motor $4:\left[\begin{array}{l}0 \\ 0 \\ 0\end{array}\right] \quad$ Motor $5:\left[\begin{array}{c}u_{5} \\ v_{5} \\ w_{5}\end{array}\right] \quad$ Motor $7:\left[\begin{array}{c}0 \\ v_{7} \\ w_{7}\end{array}\right]$

The first phase of the calibration process starts with positioning the hand attachment of the SPIDAR at some four positions called "exciting positions." Each position produces three data: the lengths of the three strings:

- Position 1 (Motor5) gives $l_{45}, l_{25}$ and $l_{57}$,

- Position 2 (Motor 4 ) gives $l_{42}, l_{47}$ and $l_{45}$,

- Position 3 (Motor2) gives $l_{42}, l_{25}$ and $l_{27}$,

- Position 3 (Motor7) gives $l_{47}, l_{27}$ and $l_{57}$.

where $l_{\mathrm{ij}}$ is the distance between Motor $i$ and Motor $j$. These lengths provide six equations (7), where $v_{2}, v_{7}, w_{7}, u_{5}, v_{5}$ and $w_{5}$ are the six parameters to calibrate. The system is solvable and provides the following parameters (8):

These results prove that the SPIDAR can be calibrated without an external system. Simulations confirmed the correct -ness of this demonstration. Note that: (i) if the position and orientation of any motor is known in an external frame, it becomes easy to transfer the position of each motor in this new external frame, and (ii) if a high accuracy is needed, least 
square methods can be used (each length is measured at least two times: $l_{45}$ is given by positions 1 and 2).

$$
\begin{aligned}
& l_{42}{ }^{2}=v_{2}{ }^{2} \\
& l_{47}^{2}=v_{7}^{2}+w_{7}^{2} \\
& l_{45}{ }^{2}=u_{5}^{2}+v_{5}^{2}+w_{5}^{2} \\
& l_{27}^{2}=\left(y_{7}-v_{2}\right)^{2}+w_{7}^{2} \\
& l_{25}{ }^{2}=u_{5}^{2}+\left(v_{5}-v_{2}\right)^{2}+w_{5}^{2} \\
& \left(l_{75}{ }^{2}=u_{5}{ }^{2}+\left(v_{7}-v_{5}\right)^{2}+\left(w_{7}-w_{5}\right)^{2}\right. \\
& \left\{\begin{array}{l}
v_{2}=l_{42} \\
v_{7}=\frac{-l_{27}{ }^{2}+l_{17}{ }^{2}+v_{2}^{2}}{2 v_{2}} \\
w_{7}=\sqrt{l_{17}^{2}-v_{7}^{2}} \\
v_{5}=\frac{l_{45^{2}-l_{25}{ }^{2}+v_{2}{ }^{2}}}{2 v_{2}} \\
w_{5}=\frac{l_{25}{ }^{2}-l_{75}^{2}+\left(v_{7}-v_{5}\right)^{2}-\left(v_{5}-v_{2}\right)^{2}+w_{7}{ }^{2}}{2 w_{7}} \\
u_{7}=\sqrt{l_{15}{ }^{2}-v_{5}^{2}-w_{5}^{2}}
\end{array}\right.
\end{aligned}
$$

\section{D - Tactile feedback}

Finger flexion is recorded using wireless 14 DoF data-gloves from the Fifth Dimension Technology (5DT) [29]. Tactile feedback is provided to the user's hands by using small mobile phone motors attached to the glove with Velcro strips. The tactile feedback glove allows us to simultaneously use up to 10 motors positioned anywhere on the user hands. Fig. 4a shows the tactile feedback glove. A user wearing the glove is shown in Fig. 4b. Since the SPIDAR measures only position, a Polhemus tracking sensor is used for orientation tracking [30].

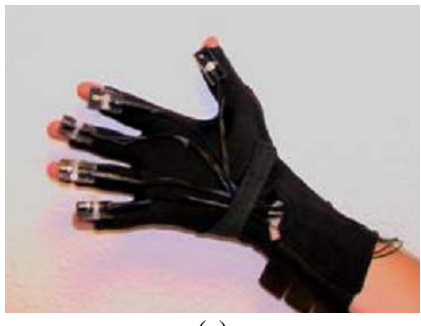

(a)

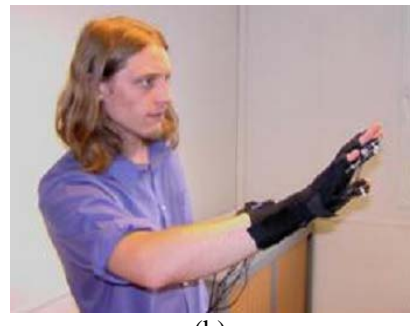

(b)
Fig. 4: (a) A 5DT data glove equipped with vibro-tactile motors; (b) operator wearing the glove.

\section{PRODUCT DESIGN APPLICATION}

\section{A - Description}

To illustrate the suitability of our human-scale VE for product design, two industrial applications were developed. The first application involves accessibility testing using a digital car mockup. The SPIDAR allows the user to gain access to the different parts of the mockup (Fig. 5). Collision feedback is provided through visual change (color) of the virtual hand, and could be reinforced by vibro-tactile feedback (on fingertips), or auditory feedback (pitch tone). The second application involves a car-light maintenance task. Visual and auditory feedbacks are associated with collisions and correct placement of the car-lights in the mockup. Moreover, car-light weight and inertia are provided using the SPIDAR.

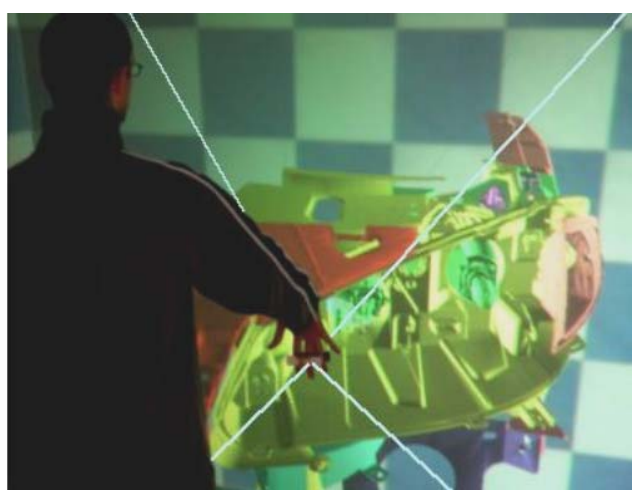

Fig. 5: Picture of a user accessing a hidden part of the virtual mock-up with the SPIDAR.

\section{B- Software environment}

Most of the applications using SPIDAR systems are developed in $\mathrm{C} / \mathrm{C}++$ language and use .wrl (VRML) or .x files that are loaded in the Springhead framework [27]. This open source software toolkit is used to handle collision detection and physical simulation. Moreover it allows high-level force control functions. However, our simulation only uses the Springhead framework to track the user's hands positions and to display the weight and inertia of the manipulated product parts. Given the high level of detail of our virtual mockup, it seemed more appropriate to use a more robust, commercial quality physics engine such as AGEIA NovodeX [31]. The choice of this physics engine was also justified by the soon commercially available first haptic dedicated processor that will allow very high performance in terms of collision detection accuracy and time processing, leading to the use of more realistic virtual models. CAD models were initially developed under the CATIA environment [32]. To integrate them into our open source software environment, these models had to be first exported into an stl file format (that is common to CATIA and 3D Studio Max), and then converted into a .3DS file format, from which it can be loaded into the simulation. The procedure, with our open source application, allows easy graphical and physical integration of any new CAD model in our multi-modal virtual environment (Fig. 6). When the graphical 3D mesh of an object is loaded, a "physical" 3D mesh is once and for all built from it, and then used for collision detection, ensuring a spatio-temporal coherence between tactual and visual feedbacks.

\section{REACH AND TOUCH EXPERIMENT}

A-Aim

Accessing certain parts of the model can sometimes be diffi 
The International Journal of Virtual Reality, 2006, 5(2):37-44

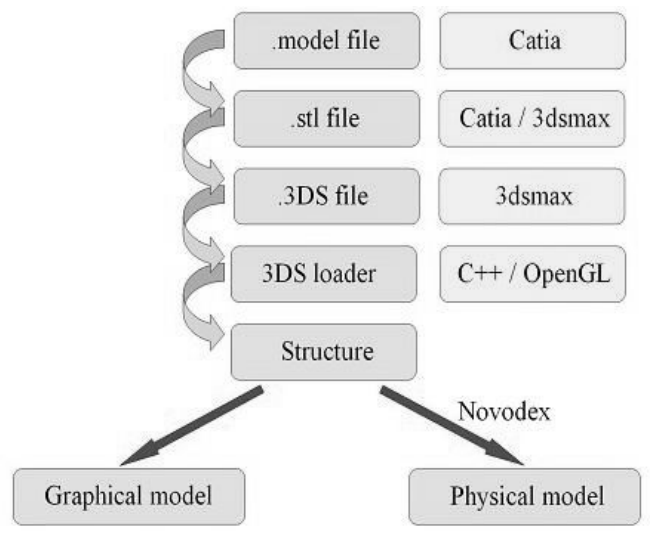

Fig. 6: Illustration of the CAD to VR procedure used for the construction of graphical and physical models.

-cult due to the restricted visibility and physical accessibility. Thus, visual feedback from the virtual hand is not always available, as the user has to reach for a hidden part of the mock-up. In such case, other sensory cues such as auditory, tactile or kinesthetic ones are welcome. The purpose of this experiment was twofold: (1) to examine the effect of vibro-tactile feedback (transmitted through the glove) on user performance (completion time) in a task involving reach-and-touch of different parts of the digital mock-up, and (2) to investigate the use of sensory substitution, i.e. vibro-tactile information transmitted though visual and auditory signals in such task.

\section{B-Method}

Five right-handed volunteer subjects participated in this experiment. They were asked to complete the task illustrated in Fig. 7, as quickly as possible. It consisted in sequentially reaching and touching parts 1 (Fig. 7a), 2 (Fig. 7b), 3 and 4 (Fig. 7c), without penetrating the mock-up. Part 3 had to be reached and touched from the back of the mock-up. In this case, visual feedback (i.e. the virtual hand) was not always available. Each subject performed the task in the following conditions: no sensory feedback («None» condition), visual feedback («Visual» condition), auditory feedback («Auditory» condition), and tactile feedback («Tactile» condition), in a random order.

This was done to avoid any learning effect between conditions. In all conditions visual feedback from the mock-up was always available. Five trials were done for each condition. In the «None» condition, no feedback was available to the user. When a collision between the virtual hand and the digital mock-up occurred, either the color of the virtual hand turned to red («Visual» condition), or an auditory signal was displayed («Auditory» condition). In the «Tactile» condition, a tactile cue was displayed on the user's fingertips using the vibro-tactile feedback glove. In all conditions, the SPIDAR was only used for hand tracking (no force feedback cues were displayed).

This was done to isolate vibro-tactile information in the «Tactile» condition. Subjects stood in front of the $2 \mathrm{~m} \times 2.5 \mathrm{~m}$ rear-projected screen at the center of the SPIDAR workspace. In all conditions, the tactile feedback glove was fitted to their right hand.

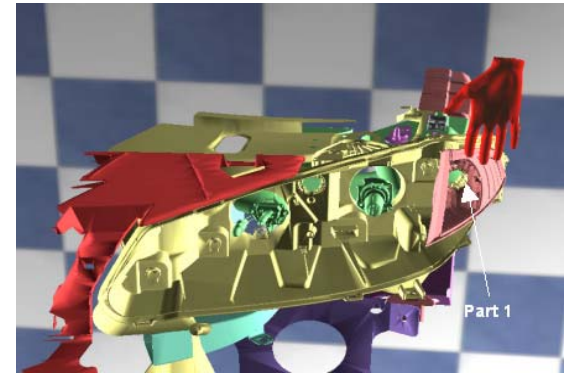

(a)

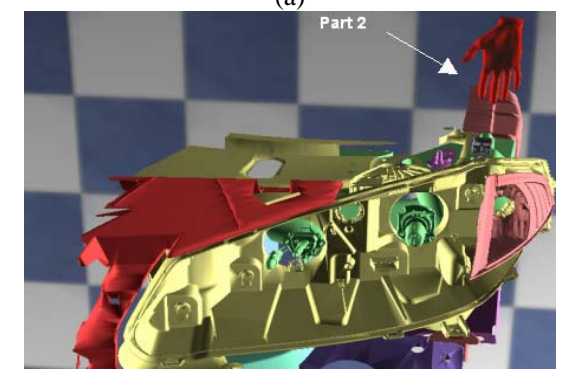

(b)

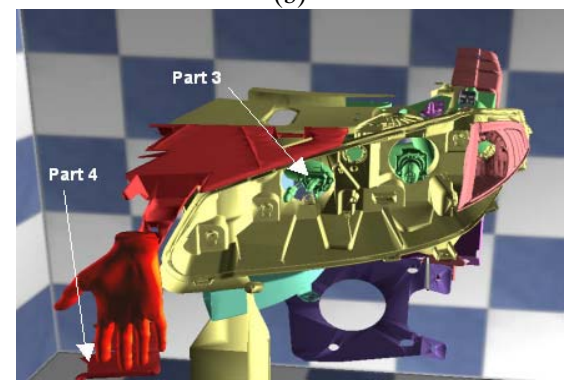

(c)

Fig. 7: Steps of the experimental task: reach and touch (a) part 1, (b) part 2, (c) part 3 and 4 . Part 3 has to be accessed from the back of the mock-up. See Color Plate 20

\section{C-Results}

In order to assess user performance, task completion time was recorded for each single trial. The data was analyzed using a one-way analysis of variance (ANOVA). Results, illustrated in Fig. 8, revealed that sensory feedback has a significant effect on task completion time: $\mathrm{F}(4,3)=7.88 ; \mathrm{p}<0.005$. A statistical difference between the «None» condition and the «Visual», «Auditory» and «Tactile» condition respectively was observed. In the open loop case («None» condition), the average completion time was $18.64 \mathrm{sec}(\mathrm{STD}=0.66)$. However, when sensory feedback was available, average completion time was $14.94 \mathrm{sec}(\mathrm{STD}=0.66$ ) for auditory feedback, $15.68 \mathrm{sec}$ (STD $=0.66)$ for visual feedback, $14.56 \mathrm{sec}(\mathrm{STD}=0.66)$ for tactile feedback. Thus, auditory and tactile feedback improves subjects' performance by about $25 \%$ compared to no feedback. Observation during the task revealed that operators had more difficulties in reaching and touching the occluded part (part 3). Subjects reported that tactile feedback provided a "feeling of touch" and therefore an efficient cue for collision with the digital mock-up. Some subjects reported that auditory feedback, although not providing tactile cues, provides an intuitive and efficient substitute for tactual feedback. 


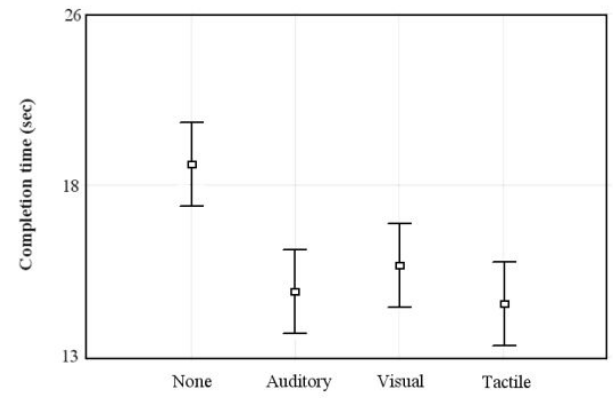

Fig. 8: Mean task completion time vs. sensory feedback.

\section{MAINTENANCE EXPERIMENT}

\section{A-Aim}

The purpose of this experiment was to investigate the effect of visual and auditory feedbacks on operator performance (task completion time and placement accuracy) in a car-light maintenance task. Objects weight and inertia were rendered using the SPIDAR. This was done to increase realism of the task. Operators had to remove a set of three car-lights and replace them by a new set. Fig. 9 shows the manipulation workspace at the beginning (see Fig. 9(a)) and at the end of the task (see Fig. 9(b)).

\section{B-Method}

18 right-handed volunteer subjects participated in this experiment. They were divided in 3 groups of 6 subjects each. Each group of subjects performed in the following conditions: no sensory feedback («None» condition), visual feedback («Visual» condition), auditory feedback («Auditory» condition). These feedbacks are associated with correct placement of the light in the mock-up. In order to avoid any training transfer, the conditions were counterbalanced and 10 single trials were performed for each condition. As in the previous experiment, in the «None» condition, no sensory feedback was available to the user. In this experiment, subjects were seated in front of the $2 \mathrm{~m} \times 2.5 \mathrm{~m}$ rear-projected screen about at the center of the SPIDAR workspace. Subjects were asked to complete the task illustrated in Fig. 9. This task consisted in removing a set of three car-lights (lights $1 \mathrm{~b}, 2 \mathrm{~b}$, and $3 \mathrm{~b}$ ) and replaced it by a new set of lights (lights $1 \mathrm{a}, 2 \mathrm{a}$, and 3a) as quickly and accurate as possible. A control timer was used to record task completion time. Users had to activate/deactivate it at the beginning and at the end of the task. As shown in Fig. 10b, car-lights were be manipulated using a spherical 3D cursor. When a collision between the 3D cursor and a car-light occurred, the color of the light slightly became darker for selection feedback. As soon as the light collided the mock-up, it turned from light brown to red. When correctly placed (within distance threshold) on the virtual mock-up, either lights turned to green («Visual» condition) (see Fig.10 (a)) or a sound was emitted («Auditory» condition). As illustrated in Fig.10(b), all lights had to be placed from the back of the virtual mock-up. As soon as all lights were correctly placed, subjects had to push on the control timer.

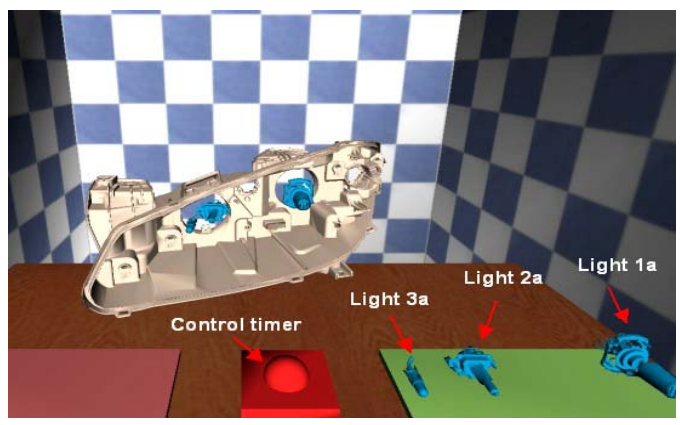

(a)

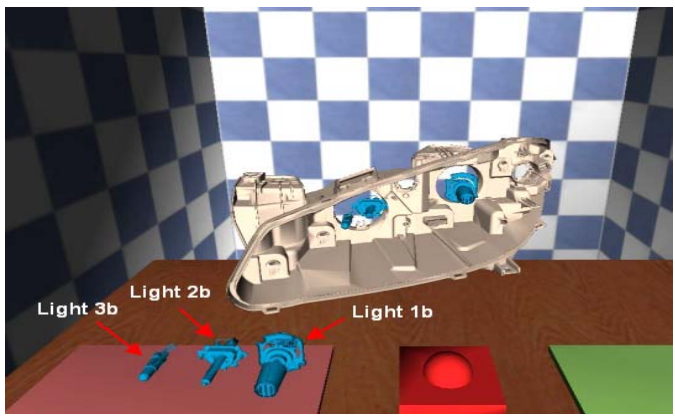

(b)

Fig. 9: Manipulation workspace: (a) beginning of the task (lights 1a, 2a and 3a are correctly placed), (b) end of the task lights (1b, 2b and 3b are correctly placed). See Color Plate 22

\section{$C-$ Results}

In order to assess user performance, both task completion time and placement accuracy were recorded. The data was analyzed using a one-way analysis of variance (ANOVA). Results revealed that sensory feedback have no significant effect on task completion time $(\mathrm{F}(5,2)=0.04$; $\mathrm{p}<0.96)$. Average task completion time was about $63.5 \mathrm{sec}(\mathrm{STD}=16.9)$ for the «None» condition, about $62.0 \mathrm{sec}(\mathrm{STD}=12.3)$ for the «Visual» condition, and about $64.0 \mathrm{sec}(\mathrm{STD}=15.6)$ for the «Auditory» condition. However, we observed (Fig. 11) that both visual and auditory feedbacks do have a significant effect on placement accuracy $(F(5,2)=27.80 ; p<0.005)$. In the open loop case («None» condition) average placement accuracy was about $2.8 \mathrm{~cm}$ (STD = 0.67). When sensory feedback was available, average placement accuracy was about $1.4 \mathrm{~cm}$ (STD $=0.14)$ for visual feedback, and $1.2 \mathrm{~cm} \quad(\mathrm{STD}=0.2)$ for auditory feedback. Thus, visual and auditory feedbacks improve average subjects' performance by about 54\% and 60\% respectively compared to the open loop case.

\section{CONCLUSION}

The work presented in this article is open to several theoretical and applicative research issues. In the near future, we will work on two directions: (1) full integration of the Novodex physic en -gine with the SPIDAR environment and (2) design and human 
The International Journal of Virtual Reality, 2006, 5(2):37-44

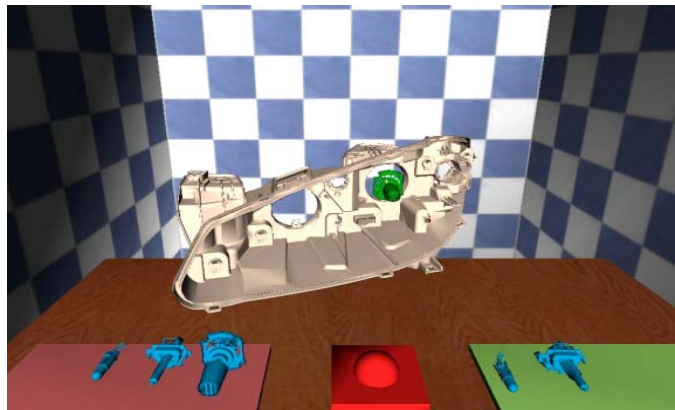

(a)

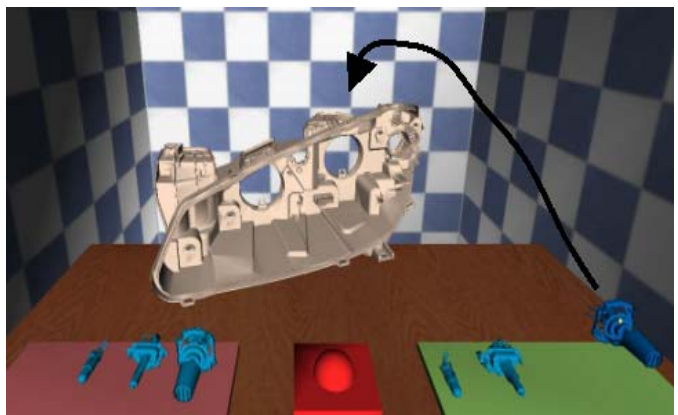

(b)

Fig. 10: Snapshot of the manipulation workspace during the task: (a) light 1a turns to green when correctly placed in the virtual mock-up, (b) orange arrows illustrates a possible trajectory for correctly placing light 1a. See Color Plate 23.

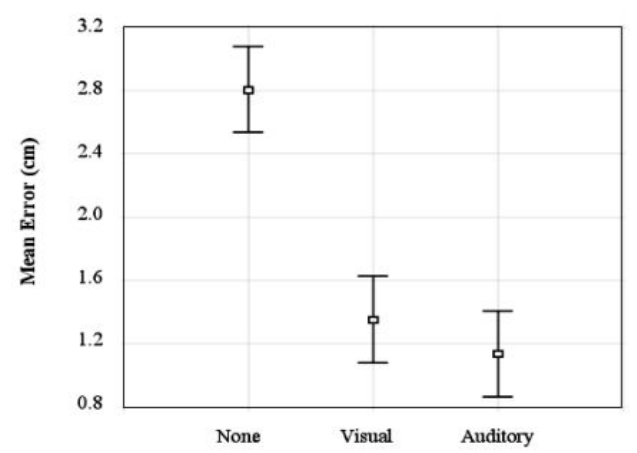

Fig. 11: Mean placement error vs. sensory feedback.

performance evaluation in task involving multi-modal interaction and multi-sensory feedbacks in both applicative and theoretical contexts. The main features of the proposed VE are the ability to display different aspects of force feedback associated mainly with contact, weight, and inertia in a relatively large workspace. Another distinguishing characteristic of the human-scale VE is that the operator does not think in terms of manipulating an input device, instead he has a full and direct use of his hands. However, the system has some drawbacks. Mainly, the strings may interfere with each other if the operator tries to turn her/himself around or fully cross his hands. Actually, this is inevitable for any system using direct contact attachment with the operator to generate force feedback. Another problem occurs when the operator moves her/his hands with a very high speed. This kind of movement makes the string no longer straight and causes a length miscalculation, which affects the precision of the hand position.

\section{REFERENCES}

[1] http://www.fakespace.com/

[2] G. Burdea, P. Richard and P. Coiffet. Integration of multi-modal I/Os for Virtual Environments, Int. J. of Human-Computer Interaction (IJHCI) Special Issue on Human-Virtual Environment Interaction, no.1, pp.5-24, 1996

[3] S. J. Biggs, M. A. Srinivasan. Haptic Interfaces, In: Stanney K. M. (ed) Handbook of the Virtual Environments: Design, Implementation and Applications, Lawrence Erlbaum Associates, Pub., London, Ch. 5, pp. 93-116,2002

[4] M. A. Srinivasan. Haptic Interfaces, In: Durlach N.I, Mavor A. (eds) Virtual Reality: Scientific and Technical Challenges, National Academic Press, Washington DC, pp. 161-187, 1995

[5] M. A. Srinivasan, C. Basdogan. Haptics in Virtual Environments: Taxonomy, Research status, and Challenges, Computers \& Graphics vol.21, no.4, pp. 393-404, 1997

[6] http://www.sensable.com/

[7] P. Richard, G. Burdea and P. Coiffet. Human performance in tasks involving virtual objects with force feedback, Proceedings of Interface to Real and Virtual Worlds Conference, Montpellier, France, pp.229 $-238,1993$

[8] P.Richard and P.Coiffet. Dextrous haptic interaction in Virtual Environments : human performance evaluation, Proceedings of the $8^{\text {th }}$ IEEE Int. Work. on Robot and Human Interaction, (RO-MAN'99), October 27-29, Pisa, Italy,pp.315-320,1999

[9] J.D.Brederson, M.Ikits, C.R.Johnson, C. D. Hansen and J. M. Hollerbach. The visual haptic workbench, Proceedings of the $5^{\text {th }}$ PHANToM Users Group Workshop, Aspen, CO, October,2000

[10] F. P. Brooks, M. Ouh-Young, J. J .Batter, P. J. Kilpatrick. Project GROPE-Haptic Display for Scientific Visualization, Computer Graphics, Proceedings of ACM SIGGRAPH 90, vol.24, no.4, pp.177185, 1990

[11] B. Grant, A. Helser. Adding Force Display to a Stereoscopic Head -Tracked Projection Display, Proceedings of the VRAIS'98, pp.81-88, 1998

[12] A. Lécuyer, C. Megard, J. M. Burkhardt, T. Lim, S.Coquillart, P.Coiffet and L.Graux. The Effect of Haptic, Visual and Auditory Feedback on an Insertion Task on a 2-Screen Workbench. Immersive Projection Technology Symposium, Orlando, US, 2002

[13] D. Borro, A. Garcia-Allonso and L. Matey. A large haptic device for aircraft engine maintainability, J. of IEEE Computer Science Society, pp.70-73, 2004

[14] Y. CAI, S. Wang and M. Sato. A Human-Scale Direct Motion Instruction System Device for Education Systems, The IEICE Transactions, E80-D 2; pp.212-217,1997

[15] W. Choi, S. -J. Jeong, N. Hashimoto, S. Hasegawa, Y. Koike and M. Sato. A Development and Evaluation of Reactive Motion Capture System with Haptic Feedback, Proceedings of the FGR'04, 37, pp. 851-856,2004

[16] M. Sato, Evolution of SPIDAR, Proceedings of the $3^{\text {rd }}$ Int. Virtual Reality Conf. (VRIC 2001), Laval, May, France, 2001

[17] N. Tarrin, S. Coquillart, S. Hasegawa, L. ouguila and M. Sato. The stringed haptic workbench: a new haptic workbench solution, Eurographics'03 vol.22, no.3, 2003

[18] S. Walairacht, M. Ishii, Y. Koike and M. Sato. Two-Handed Multi-Fingers String-Based Haptic Interface Device, The IEICE Transactions, E84-D 3; pp.365-373,2001

[19] P. Gallina, A. Rossi and R. L. Williams II. Planar cable-direct-driven robot, Part I \& II, ASME Design Technical Conferences, Pittsburgh, 2000

[20] N. Hashimoto, J. Ryu, S.-J. Jeong and M. Sato. Human-Scale Interaction with a Multi-projector Display and Multimodal Interfaces, Proceedings of the PCM'04 , vol.3, pp.23-30,2004

[21] Y. Hirata, M. Sato. 3-Dimensional Interface Device for Virtual Work Space, Proceedings of the 1992 IEEE/RSJ Int. Conf. on IROS, vol.2, pp.889-896, 1992

[22] F.-X. Inglese, S. -J. Jeong, P. Richard and M. Sato. Human-Scale Virtual Environment for Product Design, Proceedings of Virtual Concept'05, Biarritz, France, Nov.,2005

[23] M. Ishii, M. Sato. 3D Spatial Interface Device Using Tensed Strings, Presence:Teleoperators and Virtual Environments, vol.3, no.1,pp. 81-86, 1994 
[24] S. Kim, Y. Hasegawa, M. Koike and M. Sato. Tension based 7 DOF force feedback device: SPIDAR-G, Proceedings of the IEEE Virtual Reality, 2002

[25] R. L. Williams II. Planar Cable-Suspended Haptic Interface: Design for Wrench Exertion, Proceedings of the 1999 ASME Design Technical Conferences, $25^{\text {th }}$ Design Automation Conference, Las Vegas, 1999

[26] A. Paljic, N. Tarrin, S. Coquillart, L. Bouguila, M. Sato. The Passive Stringed Haptic Spidar for the Worlkbench, Eurographics'04, Grenoble, France, 2004

[27] http://springhead.info/

[28] http://www.maxonmotor.com/dc_motor.asp

[29] http://www.5dt.com

[30] http://www.polhemus.com/

[31] http://www.ageia.com/

[32] http://www.3ds.com/products-solutions/plm-solutions/catia

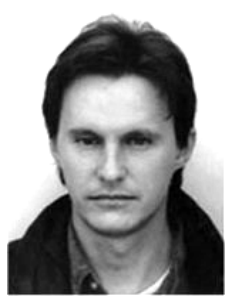

Paul RICHARD was born in Négrepelisse, France, in 1964. He received his BS and MS in Electronics and Automation from the University of Nice Sophia-Antipolis in 1990 and 1991 respectively. He received a PhD in Robotics from the University of Paris VI in 1996. Dr. Richard is currently assistant Professor at the University of Angers - ISTIA (Institut des Sciences et Techniques de l'Ingénieur d'Angers) and responsible for the virtual reality research activities of the Laboratoire d'Ingénierie des Systèmes Automatisés (LISA). For more than a decade his research has focused on virtual reality, multi-modal interaction and human performance in virtual environments. Dr. Richard was Co-Chair of VRIC (Virtual Reality International Conference) held in LAVAL in 2001, 2002 and 2003 and Co-Editor.

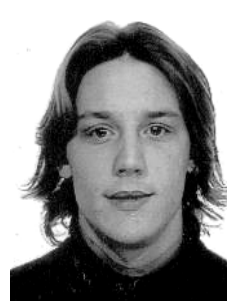

Damien CHAMARET was born in Laval, France in 1982. He is currently a phD student at Laboratoire d'Ingénierie des Systèmes Automatisés (LISA). He received a BS in Innovation Technology from the University of Angers (ISTIA) in 2004 and a MS in virtual reality from the University of Maine, France in 2005. His research activities and interests are virtual reality, multi-modal interaction, and product design.

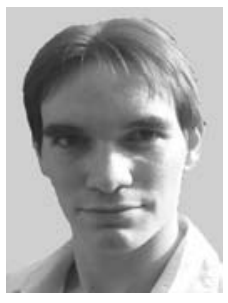

François-Xavier INGLESE was born in Nantes, France in 1978. He is currently a phD student at Laboratoire d'Ingénierie des Systèmes Automatisés (LISA). He received a BS in computers sciences from the University of Angers in 2001 and a MS in virtual reality from the University of Maine, France in 2002. His research activities and interests are virtual reality, human-virtual environments interaction and game programming.

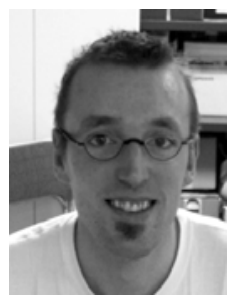

Philippe LUCIDARME was born in Sainte Catherine, France, in 1976. He received his BS and MS in Electronics and Automation from the University of Lille in 1999 and 2000 respectively. He received a $\mathrm{PhD}$ in Robotics from the University of Montpellier in 2003. Dr. Lucidarme is currently assistant Professor at Angers Institute of Technology and responsible for the robotics research activities of the Laboratoire d'Ingénierie des Systèmes Automatisés (LISA) in Angers. His research activities and interests are virtual reality and robotics. He was recently honoured by the PC104 consortium; he received the price of the best design based on PC104 for its research activities.

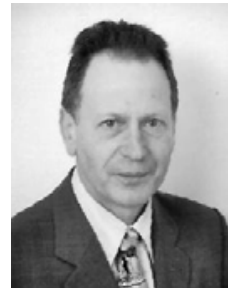

Jean-Louis FERRIER was born in Couiza, France, in 1947. He received the diploma of engineer from INSA Toulouse in 1969 and the PhD from the University of Angers in 1986. He spent two years as a control systems engineer at Motorola automotive division. $\mathrm{He}$ is currently a full Professor at the university of Angers ISTIA (Institut des Sciences et Techniques de l'Ingénieur d'Angers) and responsible for the International relations of this Institute. He was the Head of the Electrical and Computer Engineering Department of ISTIA from 1989 to 1998. From 2000 to 2002, he was the Director of the graduate school of Angers - gathering the University $\mathrm{PhD}$ students. In 2002-2003 he was appointed as a Delegate of the French Ministry of Research and Technology. He is currently the Head of the LISA whose topics are Control Engineering, Virtual Reality, Signal and Image processing. For more than a decade his research has focused on many areas, including Automatic control, Computer engineering, Automation, Virtual reality. 